\title{
Article
}

\section{Dothiorella species associated with woody hosts in Italy}

\section{Dissanayake AJ ${ }^{1,2}$, Camporesi $\mathrm{E}^{3}$, Hyde $\mathrm{KD}^{2,4}$, Phillips AJL ${ }^{5}, \mathrm{Fu} \mathrm{CY}^{1}$, Yan $\mathrm{JY}^{1}$ and $\mathrm{Li} \mathrm{XH}{ }^{1, *}$}

\footnotetext{
${ }^{1}$ Institute of Plant and Environment Protection, Beijing Academy of Agriculture and Forestry Sciences, Beijing 100097, People's Republic of China

${ }^{2}$ Center of Excellence in Fungal Research, School of Science, Mae Fah Luang University, Chiang Rai 57100, Thailand

3 A.M.B. Gruppo Micologico Forlivese "Antonio Cicognani", Via Roma 18, Forlì, Italy

${ }^{4}$ Key Laboratory for Plant Diversity and Biogeography of East Asia, Kunming Institute of Botany, Chinese Academy of Sciences, Kunming 650201, People's Republic of China

${ }^{5}$ UCIBIO-REQUIMTE, Departamento de Ciências da Vida, Faculdade de Ciências e Tecnologia, Universidade Nova de Lisboa, 2829-516 Caparica, Portugal

* Corresponding author: Xinghong Li (lixinghong1962@163.com)
}

Dissanayake AJ, Camporesi E, Hyde KD, Phillips AJL, Fu CY, Yan JY , Li XH 2016 - Dothiorella species associated with woody hosts in Italy. Mycosphere 7(1), 51-63, Doi 10.5943/mycosphere/7/1/6

\begin{abstract}
Dothiorella species are pathogens of many woody hosts but their host distribution is poorly known. In this study, a collection of Dothiorella isolates obtained from different woody hosts, including Acer, Cornus, Coronilla, Crataegus, Euonymus, Fraxinus, Laburnum, Laurus, Paliurus, Prunus, Salix, Sambucus and Ulmus species in Italy were identified. Morphological and cultural characteristics, as well as ITS and EF1- $\alpha$ sequence data, were used to identify the isolates. The results show the diversity of Dothiorella species associated with twigs and branches of these woody hosts. Four species were identified. Dothiorella sarmentorum was the dominant species $(65 \%$ of isolates), followed by D. symphoricarposicola (20\%), D. iberica (8\%) and D. vidmadera (8\%). A host-fungus index for all Dothiorella species with available molecular data from the literature is provided.
\end{abstract}

Key words - Botryosphaeriaceae - EF1- $\alpha$ - Hosts - ITS - Multigene phylogeny

\section{Introduction}

Dothiorella belongs in the family Botryosphaeriaceae, order Botryosphaeriales, and class Dothideomycetes (Wijayawardene et al. 2014) and has been the subject of several recent publications (Adesemoye et al. 2014, Chen et al. 2014, Li et al. 2014, Liu et al. 2012, Lynch et al. 2014, Pitt et al. 2015). Species of Dothiorella, like other members of this family, are pathogens, endophytes and saprobes of a wide range of woody hosts (Crous et al. 2006, Hyde et al. 2013, 2014, Liu et al. 2012, Phillips et al. 2013); however, it is not clear whether species have narrow or wide host distributions. The genus encompassed a wide range of morphologies from thin-walled, hyaline, aseptate conidia to thick-walled, brown, 1-septate conidia (Phillips et al. 2005). Sivanesan (1984) confused the taxonomy of Dothiorella by treating Dothiorella pyrenophora Sacc. (1880) as a synonym of Dothichiza sorbi Lib., which has small, hyaline, aseptate conidia and is the asexual morph of Dothiora pyrenophora (Fr.) Fr. However, Sutton (1977) clarified the taxonomic history 
by placing Dothiora pyrenophora as a homonym of Dothiorella pyrenophora. Crous \& Palm (1999) studied the holotype of $D$. pyrenophora and concluded that Dothiorella is a synonym of Diplodia. Based on morphology and molecular data, Phillips et al. (2005) revived Dothiorella for species with conidia that become brown and 1-septate, while they are still attached to the conidiogenous cells. Sexual morphs of Dothiorella have pigmented, 1-septate ascospores (Phillips et al. 2005, 2013). With the exception of $D$. sarmentorum (Fr.) Phillips, Alves \& Luque and D. iberica Phillips, Alves \& Luque, the sexual morphs of Dothiorella species are rarely encountered in nature and have never been produced in culture (Phillips et al. 2013).

Although there are 350 species records in Dothiorella, Phillips et al. (2013) revealed that cultures are available for only 17 species and of those four species have yet to be named. Abdollahzadeh et al. (2014) introduced three species names for these un-named taxa i.e. D. parva Abdollahz., Zare \& A.J.L Phillips, D. prunicola A.J.L. Phillips \& Abdollahz. and D. striata A.J.L. Phillips \& Abdollahz. Presently, 23 species are accepted in the genus (Abdollahzadeh et al. 2014, Li et al. 2014, Phillips et al. 2013, Pitt et al. 2013, 2015, Slippers et al. 2014). All species, except $D$. sarmentorum, have been introduced since 2005.

A collection of Dothiorella isolates was obtained from branches of various woody hosts in Forlì-Cesena Province, in Italy. The aim of this study was to name these species and reveal the distribution of species on the hosts. Isolates were characterised in terms of morphology and their phylogenetic position in Dothiorella. A host index for Dothiorella species based on literature records with molecular identifications is provided.

\section{Material and methods}

\section{Sample collection, specimen examination and isolations}

Specimens were collected from woody branches of Acer L., Cornus L., Coronilla L., Crataegus L., Euonymus L., Fraxinus L., Laburnum Fabr., Laurus L., Paliurus Mill., Prunus L., Salix L., Sambucus L. and Ulmus L. species in Italy. Specimens were observed and examined with a Motic SMZ 168 stereomicroscope. Micro-morphological characters were determined with a Nikon ECLIPSE 80i compound microscope and images were captured with a Canon EOS 550D digital camera. Observations and photographs were made from materials mounted in water. Measurements were made with the Tarosoft (R) Image Frame Work and images used for figures were processed with Adobe Photoshop CS3 Extended version 10.0. Single spore isolations were prepared following the method of Chomnunti et al. (2014). Spore germination on water agar (WA) was examined after $24 \mathrm{~h}$ and germinating spores were transferred to potato dextrose agar (PDA) media. Cultures were incubated at $18^{\circ} \mathrm{C}$ in the dark and cultural characteristics such as mycelium colour, shape, texture and growth rate were determined. Herbarium specimens are deposited in the Mae Fah Luang University Herbarium (MFLU) while, ex-type cultures are deposited at the Mae Fah Luang University Culture Collection (MFLUCC) with duplicates in the Kunming culture collection, China (KUMCC) and GuiZhou Culture Collection, China (GZCC).

\section{DNA extraction, PCR amplification and sequencing}

Genomic DNA was extracted from 10 day-old pure cultures grown on PDA, following a modified protocol of Armaleo \& Clerc (1995). Amplification and sequencing of the internal transcribed spacer (ITS) region and part of the translation elongation factor (EF1- $\alpha$ ) gene were performed as described by Dissanayake et al. (2015) using primer pairs ITS1 and ITS4 (White et al. 1990) and EF1-728F and EF1-986R (Carbone \& Kohn 1999), respectively.

\section{Phylogenetic analyses}

All new sequences generated in this study were checked manually and nucleotides at ambiguous positions clarified with sequences from both strands. All reference sequences were obtained from GenBank based on previously published study by (Hyde et al. 2014) as listed in Table 1. Combined datasets of ITS and EF genes were aligned using MAFFT v.7 (Katoh \& Toh 
2008, http://mafft.cbrc.jp/alignment/server/). The alignment was checked and improved manually where necessary using BioEdit (Hall 2006). Maximum-parsimony (MP) analysis was performed using PAUP v. 4.0b10 (Swofford 2003) to obtain the most parsimonious tree. Ambiguously aligned regions were excluded and gaps were treated as missing data. Trees were inferred using the heuristic search option with Tree Bisection Reconnection (TBR) branch swapping and 1000 random sequence additions. Maxtrees were set up to 1000 and branches of zero length were collapsed and all multiple parsimonious trees were saved. Descriptive tree statistics for parsimony such as Tree Length (TL), Consistency Index (CI), Retention Index (RI), Relative Consistency Index (RC) and Homoplasy Index (HI) were calculated. Clade stability was evaluated by 1000 bootstrap replications resulting from maximum parsimony analysis (Hillis \& Bull 1993). The best model of evolution for each gene region was determined by using MrModeltest 2.2 (Nylander 2004). The general time-reversible model of evolution (Rodriguez et al. 1990), including estimation of invariable sites and assuming a discrete gamma distribution with six rate categories $(\mathrm{GTR}+\mathrm{I}+\mathrm{G})$ was used for BI analyses for ITS and EF1- $\alpha$ sequence datasets. Posterior probabilities (PP) were determined by Markov Chain Monte Carlo sampling (BMCMC) in MrBayes v. 3.0b4 (Ronquist \& Huelsenbeck 2003). Four simultaneous Markov chains were run for 1000000 generations and trees were sampled every 100th generation and 10000 trees were obtained. The first 2000 trees, representing the burn-in phase of the analyses, were discarded while remaining 8000 trees were used for calculating posterior probabilities in the majority rule consensus tree (critical value for the topological convergence diagnostic set to 0.01) (Crous et al. 2006). Phylogenetic trees were drawn using Treeview v. 1.6.6 (Page 1996).

\section{Results}

\section{DNA phylogeny}

The combined ITS and EF1 $-\alpha$ dataset consisted of 727 characters (including alignment gaps) for 37 ingroup and 1 outgroup taxa. Of the 727 characters 480 were constant and 56 were variable and parsimony uninformative. Maximum parsimony analysis of the remaining 91 parsimonyinformative characters resulted in 309 equally parsimonious trees $(\mathrm{CI}=0.673, \mathrm{RI}=0.735, \mathrm{RC}=0.495$, $\mathrm{HI}=0.327$ ) and the best scoring first MP tree is shown in Fig. 1. The Dothiorella isolates studied were distributed into four sub-clades in the phylogenetic analysis (Fig. 1). The three isolates from Laburnum alpinum, Laurus nobili and Sambucus nigra were identified as Dothiorella symphoricarposicola (Fig. 3); one isolate from Acer opalus was identified as Dothiorella iberica and a set of isolates from Cornus sanguinea, Coronilla emerus, Crataegus sp., Euonymus europaeus, Paliurus spina-christi, Prunus dulcis, Salix sp. and Ulmus minor clustered in a larger group identified as D. sarmentorum (Fig. 2). The remaining clade containing isolates from Fraxinus ornus represents Dothiorella vidmadera.

\section{Discussion}

In this study Dothiorella isolates obtained from different woody hosts, including Acer, Cornus, Coronilla, Crataegus, Euonymus, Fraxinus, Laburnum, Laurus, Paliurus, Prunus, Salix, Sambucus and Ulmus species in Italy were identified and provide additional information on the distribution of Dothiorella species on various hosts. The 23 Dothiorella species along with their known hosts, host families, orders and their distribution are presented in Table 2. Dothiorella iberica has been isolated from 12 woody hosts world-wide (Phillips et al. 2005, 2008, Lynch et al. 2013, 2014, Luque et al. 2005, Burgess et al. 2006, Zhou et al. 2010, Wet et al. 2009); and this study provides the first report of D. iberica isolated from Acer opalus (Table 2). Phillips et al. (2013) reported that Dothiorella sarmentorum is a cosmopolitan species and has been isolated from 34 different host species including Malus, Menispermum, Prunus, Pyrus and Ulmus. However, molecular studies have proven Dothiorella sarmentorum is described in only 17 woody hosts. Our study reveals the isolation of $D$. sarmentorum from five woody hosts in Italy and this is the first report of $D$. sarmentorum from these woody hosts (Table 2). An additional three isolates 


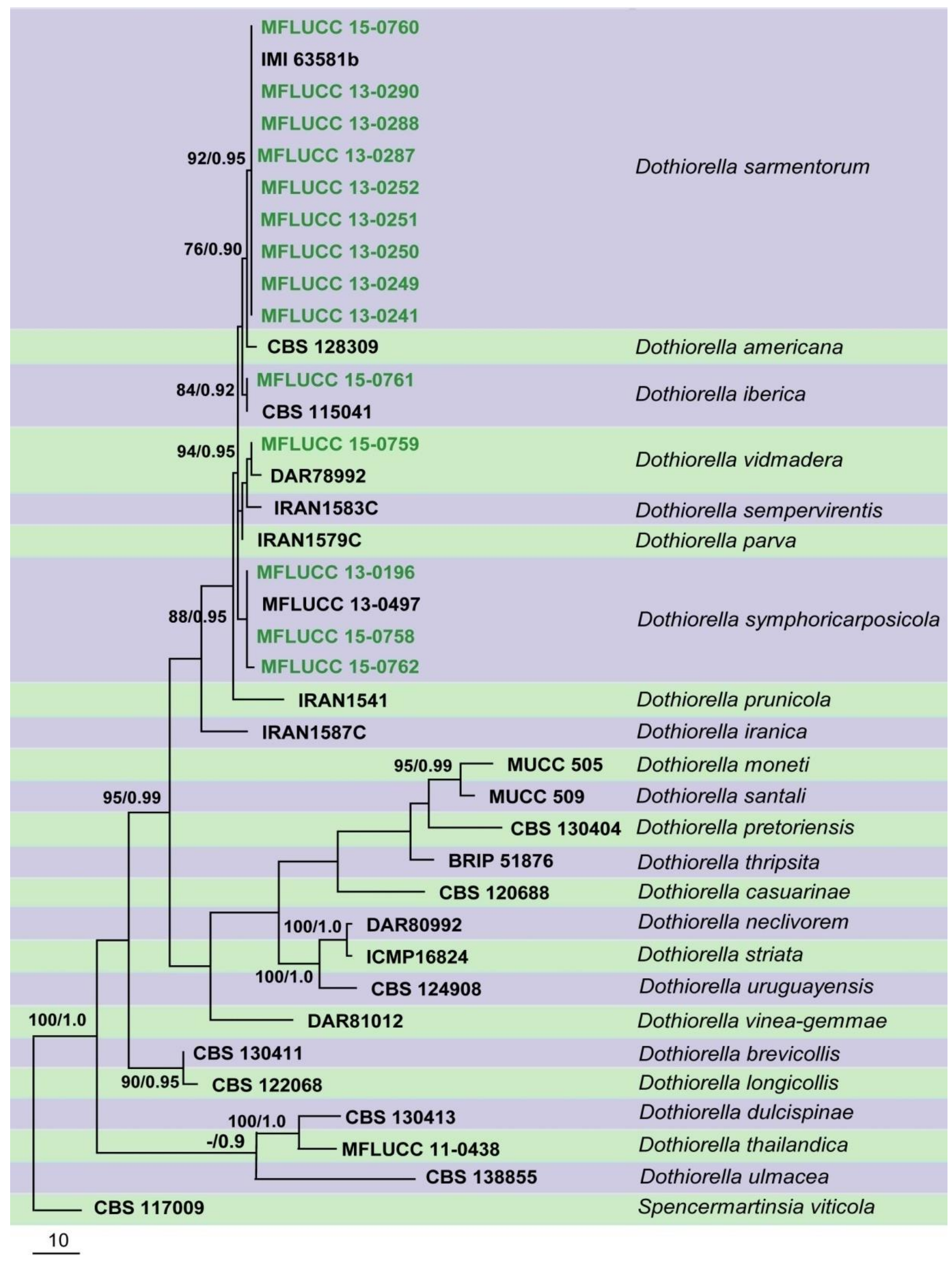

Fig. 1 - One of 309 most parsimonious trees obtained from combined ITS and EF-1 $\alpha$ sequence data, for all ex-types from species in Dothiorella. Maximum parsimony bootstrap values $(>70 \%)$ and Bayesian inference values $(>0.9)$ are given on the nodes. The tree is rooted to Spencermartinsia viticola (CBS 117009). 

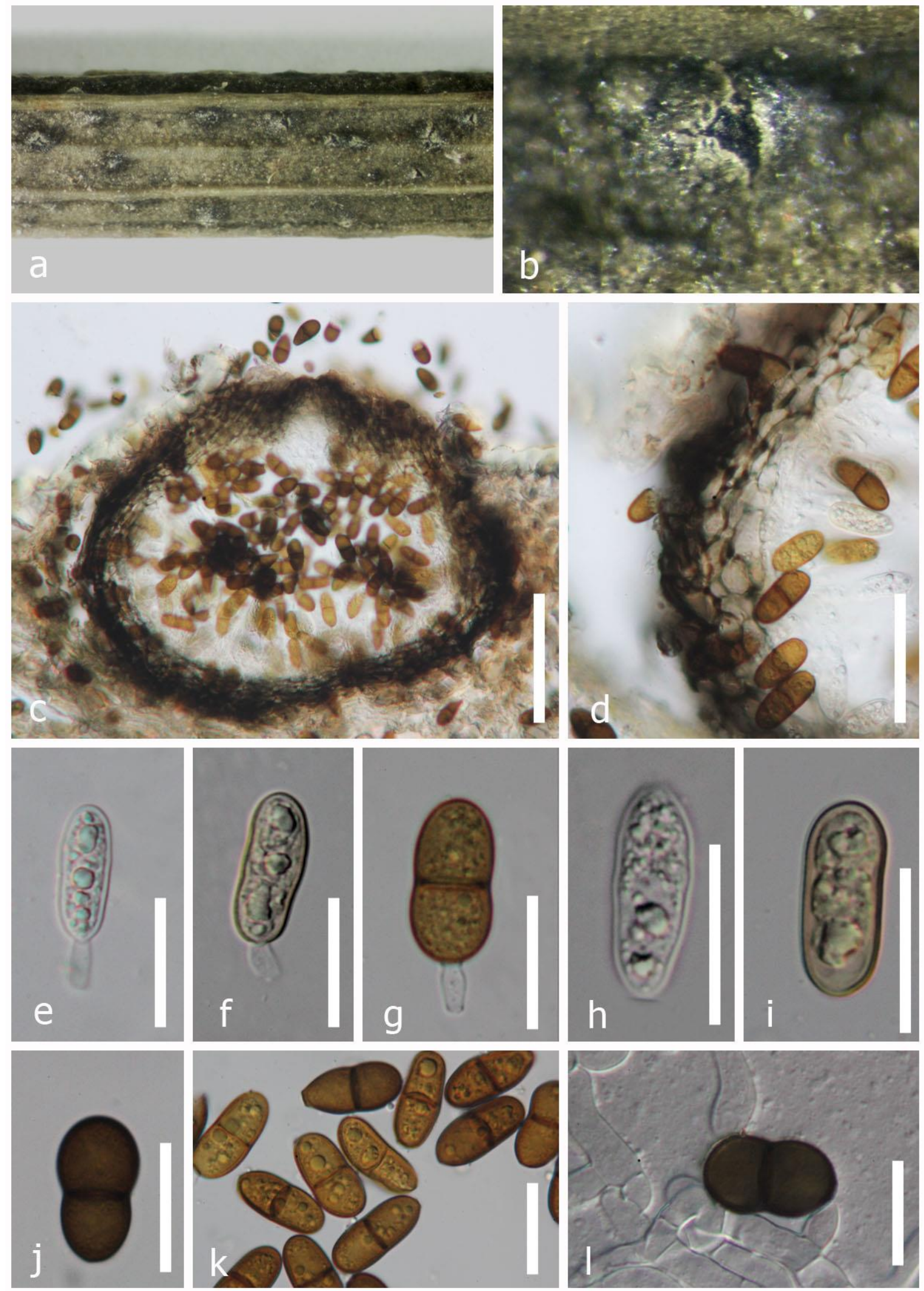

Fig. 2 - Dothiorella sarmentorum. a, b - Conidiomata on host substrate, c - Cross section of conidioma, $\mathbf{d}$ - Peridium, e, $\mathbf{f}$ - Immature conidia attached to conidiogenous cells, $\mathbf{g}$ - Mature conidia attached to conidiogenous cells, $\mathbf{h}, \mathbf{i}$ - Immature hyaline conidia, $\mathbf{j}, \mathbf{k}$ - Mature conidia, $\mathbf{l}-$ Germinating conidia. Scale bars: $c=100, d=50, e-1=20 \mu \mathrm{m}$. 

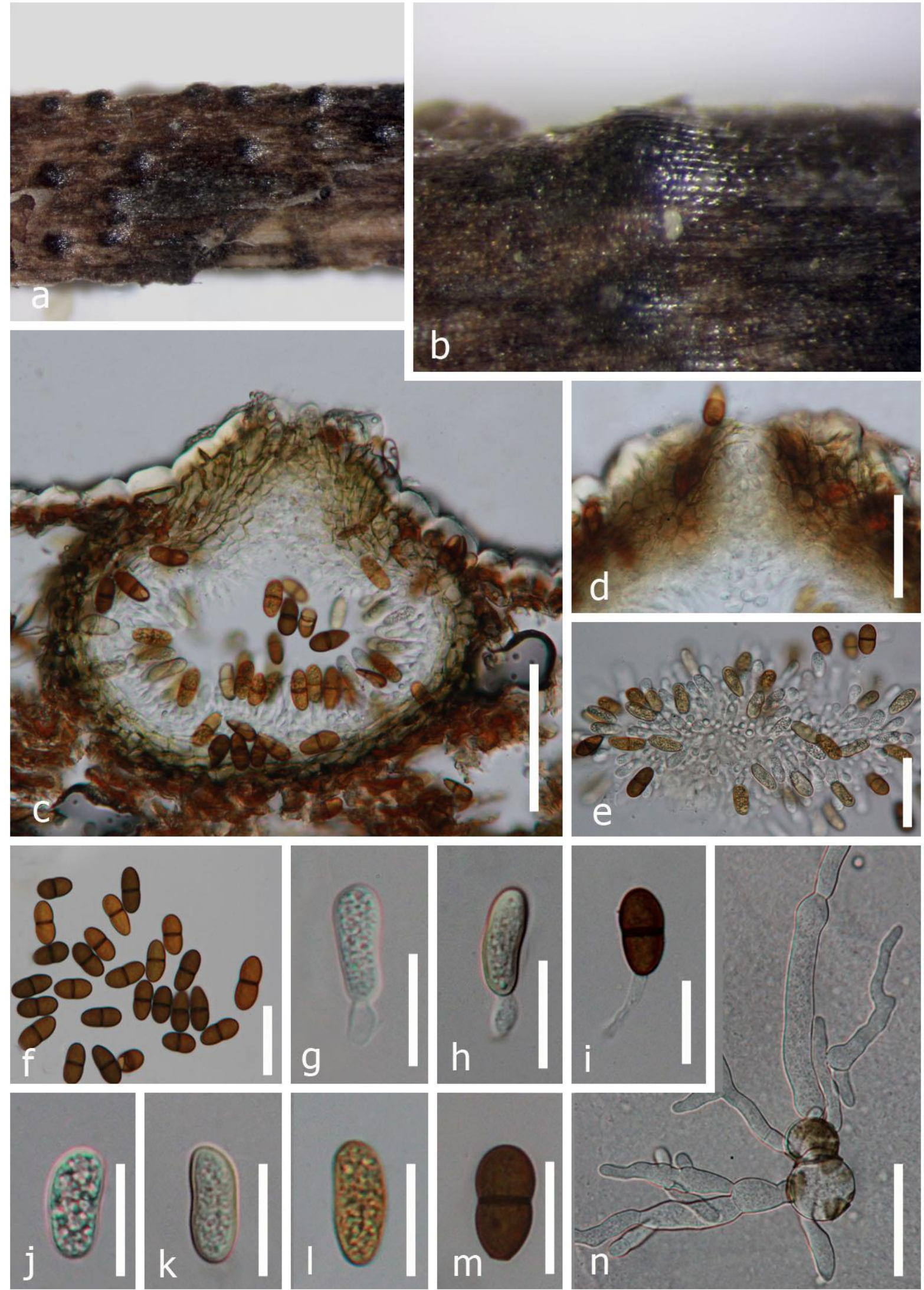

Fig. 3 - Dothiorella symphoricarposicola. a, b - Conidiomata on host substrate, $\mathbf{c}$ - Cross section of conidioma, $\mathbf{d}$ - Ostiole, $\mathbf{e}$ - Immature/mature conidia attached to conidiogenous cells, $\mathbf{f}$ - Mature conidia, $\mathbf{g}, \mathbf{h}$ - Immature hyaline conidia attached to conidiogenous cells, $\mathbf{i}$ - mature conidia attached to conidiogenous cell, $\mathbf{j}, \mathbf{k}$ - Immature hyaline conidia, $\mathbf{l}, \mathbf{m}$ - Mature conidia, $\mathbf{n}$ Germinating conidia. Scale bars: $\mathrm{c}=100, \mathrm{~d}=50, \mathrm{e}-\mathrm{f}=40, \mathrm{~g}-\mathrm{n}=20 \mu \mathrm{m}$. 
Table 1 Dothiorella isolates included in the phylogenetic analysis. Newly deposited sequences in GenBank are italicized

\begin{tabular}{|c|c|c|c|c|c|c|}
\hline \multirow[t]{2}{*}{ Species } & \multirow[t]{2}{*}{ Strain } & \multirow[t]{2}{*}{ Host } & \multirow[t]{2}{*}{ Locality } & \multirow[t]{2}{*}{ Collecter } & \multicolumn{2}{|c|}{$\begin{array}{c}\text { GenBank Accession } \\
\text { numbers }\end{array}$} \\
\hline & & & & & ITS & EF 1- $\boldsymbol{\alpha}$ \\
\hline Dothiorella americana & CBS 128309 & Vitis vinifera & Missouri, United States & K. Striegler \& G.M. Leavitt & HQ288218 & HQ288262 \\
\hline D. brevicollis & CBS 130411 & Acacia karroo & Pretoria, South Africa & F. Jami \& M. Gryzenhout & JQ239403 & JQ239390 \\
\hline D. casuarinae & CBS 120688 & Casuarina sp. & Canberra, Australia & M.J. Wingfield & DQ846773 & DQ875331 \\
\hline D. dulcispinae & CBS 130413 & Acacia karroo & Pretoria, South Africa & F. Jami \& M. Gryzenhout & JQ239400 & JQ239387 \\
\hline D. iberica & CBS 115041 & Quercus ilex & Aragón, Spain & J. Luque & AY573202 & AY573222 \\
\hline D. iberica & MFLUCC 15-0761 & Acer opalus & Forlì-Cesena, Italy & E. Camporesi & KU234781 & KU234795 \\
\hline D. iranica & IRAN1587C & Olea europaea & Golestan, Iran & A. Javadi & KC898231 & KC898214 \\
\hline D. longicollis & CBS 122068 & Lysiphyllum cunninghamii & Tunnel Creek, Australia & T.I. Burgess & EU144054 & EU144069 \\
\hline D. moneti & MUCC 505 & Acacia rostellifera & Yalgorup, Australia & K.M. Taylor & EF591920 & EF591971 \\
\hline D. neclivorem & DAR80992 & Vitis vinifera & Pokolbin, Australia & N. Wunderlich & KJ573643 & KJ573640 \\
\hline D. parva & IRAN1579C & Corylus avellana & Ardabil, Iran & J. Abdollahzadeh \& A. Javadi & КC898234 & KC898217 \\
\hline D. pretoriensis & CBS 130404 & Acacia karroo & Pretoria, South Africa & F. Jami \& M. Gryzenhout & JQ239405 & JQ239392 \\
\hline D. prunicola & IRAN1541 & Prunus dulcis & Algarve, Portugal & E. Diogo & EU673313 & EU673280 \\
\hline D. santali & MUCC 509 & Santalum acuminatum & Yalgorup, Australia & K.M. Taylor & EF591924 & EF591975 \\
\hline D. sarmentorum & IMI 63581b & Ulmus sp. & Warwickshire, England & E.A. Ellis & AY573212 & AY573235 \\
\hline D. sarmentorum & MFLUCC 15-0760 & Cornus sanguinea & Forlì-Cesena, Italy & E. Camporesi & KU234779 & $K U 234793$ \\
\hline D. sarmentorum & MFLUCC 13-0241 & Salix sp. & Forlì-Cesena, Italy & E. Camporesi & KU234780 & KU234794 \\
\hline D. sarmentorum & MFLUCC 13-0249 & Ulmus minor & Forlì-Cesena, Italy & E. Camporesi & KU234783 & $K U 234797$ \\
\hline D. sarmentorum & MFLUCC 13-0250 & Crataegus sp & Forlì-Cesena, Italy & E. Camporesi & $K U 234784$ & $K U 234798$ \\
\hline D. sarmentorum & MFLUCC 13-0251 & Euonymus europaeus & Forlì-Cesena, Italy & E. Camporesi & KU234785 & KU234799 \\
\hline D. sarmentorum & MFLUCC 13-0252 & Crataegus $s p$ & Forlì-Cesena, Italy & E. Camporesi & KU234786 & KU234800 \\
\hline D. sarmentorum & MFLUCC 13-0287 & Paliurus spina-christi & Forlì-Cesena, Italy & E. Camporesi & KU234787 & KU234801 \\
\hline D. sarmentorum & MFLUCC 13-0288 & Prunus dulcis & Forlì-Cesena, Italy & E. Camporesi & $K U 234788$ & $K U 234802$ \\
\hline D. sarmentorum & MFLUCC 13-0290 & Coronilla emerus & Forlì-Cesena, Italy & E. Camporesi & $K U 234789$ & $K U 234803$ \\
\hline D. sempervirentis & IRAN1583C & Cupressus sempervirens & Golestan, Iran & M.A. Aghajani & KC898236 & КC898219 \\
\hline D. striata & ICMP16824 & Citrus sinensis & Kerikeri, New Zealand & P.R. Johnston & EU673320 & EU673287 \\
\hline D. symphoricarposicola & MFULCC 13-0497 & Symphoricarpos sp. & Forlì-Cesena, Italy & E. Camporesi & KJ742378 & KJ742381 \\
\hline D. symphoricarposicola & MFLUCC 13-0196 & Sambucus nigra & Forlì-Cesena, Italy & E. Camporesi & $K U 234782$ & $K U 234796$ \\
\hline D. symphoricarposicola & MFLUCC 15-0762 & Laurus nobilis & Forlì-Cesena, Italy & E. Camporesi & KU234790 & KU234804 \\
\hline D. symphoricarposicola & MFLUCC $15-0758$ & Laburnum alpinum & Forlì-Cesena, Italy & E. Camporesi & KU234791 & KU234805 \\
\hline D. thailandica & CBS 133991 & Bambusa sp. & Chang Rai, Thailand & D.Q. Dai & JX646796 & JX646796 \\
\hline D. thripsita & BRIP 51876 & Acacia harpophylla & Tallegalla, Australia & D.J. Tree \& C.E.C. Tree & FJ824738 & KJ573639 \\
\hline D. ulmacea & CBS 138855 & Ulmus leavis & Germany & R.K. Schumacher & KR611881 & KR611910 \\
\hline D. uruguayensis & CBS 124908 & Hexachlamis edulis & Paysandu, Uruguay & C.A. Pérez & EU080923 & EU863180 \\
\hline D. vidmadera & DAR78992 & Vitis vinifera & Eden Valley, Australia & W.M. Pitt \& A. Loschiavo & EU768874 & EU768881 \\
\hline D. vidmadera & MFLUCC15-0759 & Fraxinus ornus & Forlì-Cesena, Italy & E. Camporesi & KU234792 & $K U 234806$ \\
\hline D. vinea-gemmae & DAR81012 & Vitis vinifera & Pokolbin, Australia & N. Wunderlich & KJ573644 & KJ573641 \\
\hline Spencermartinsia viticola & CBS 117009 & Vitis vinifera & Spain & - & KF766228 & AY905559 \\
\hline
\end{tabular}


Table 2 Distribution of Dothiorella species on host plants. Details of Dothiorella species studied in this study are in bold. Species distribution data is used only if identifications are supported by molecular data and are post-2005.

\begin{tabular}{|c|c|c|c|c|c|}
\hline Dothiorella species & Host & Family & Order & Distribution & References \\
\hline Dothiorella americana & Vitis vinifera & Vitaceae & Vitales & USA & Úrbez-Torres et al. 2012 \\
\hline D. brevicollis & Acacia karroo & Fabaceae & Fabales & South Africa & Jami et al. 2012 \\
\hline D. casuarinae & Casuarina sp. & Casuarinaceae & Fagales & Australia & Wet et al. 2009 \\
\hline D. dulcispinae & Acacia karroo & Fabaceae & Fabales & South Africa & Jami et al. 2012 \\
\hline \multirow[t]{14}{*}{ D. iberica } & Acer opalus & Sapindaceae & Sapindales & Italy & This study \\
\hline & Citrus sinensis & Rutaceae & Sapindales & California & Adesemoye et al. 2014 \\
\hline & Cupressus macrocarpa & Cupressaceae & Pinales & Algeria & Azouaoui-Idjer et al. 2012 \\
\hline & Juglans regia & Juglandaceae & Fagales & California & Chen et al. 2014 \\
\hline & Juniperus sp, & Cupressaceae & Pinales & Portugal & Alves et al. 2013 \\
\hline & Malus pumila & Rosaceae & Rosales & Spain & Phillips et al. 2005 \\
\hline & Olea europaea & Oleaceae & Lamiales & California & Úrbez-Torres et al. 2013 \\
\hline & Ostrya sp. & Betulaceae & Fagales & Italy & Pavlic-Zupanc et al. 2015 \\
\hline & Persea americana & Lauraceae & Laurales & California & Mcdonald \& Eskalen 2011, Twizeyimana et al. 2013 \\
\hline & Pistacia sp. & Anacardiaceae & Sapindales & California & Chen et al. 2014 \\
\hline & Prunus dulcis & Rosaceae & Rosales & California & Doll et al. 2015 \\
\hline & Quercus agrifolia & Fagaceae & Fagales & California & Lynch et al. 2014 \\
\hline & Quercus ilex & Fagaceae & Fagales & Spain & $\begin{array}{l}\text { Phillips et al. 2005, 2008, Lynch et al. 2013, 2015, } \\
\text { Luque et al. 2005,Burgess et al. 2006, Liu et al. 2012, } \\
\text { Zhou et al. 2010, Wet et al. } 2009\end{array}$ \\
\hline & Vitis vinifera & Vitaceae & Vitales & $\begin{array}{l}\text { Australia,California,Ne } \\
\text { w Zealand,California }\end{array}$ & $\begin{array}{l}\text { Úrbez-Torres et al. 2007, Qiu et al. 2011, Baskaratevan } \\
\text { et al. 2012, Pitt et al. 2013, 2015, Lynch et al. } 2013\end{array}$ \\
\hline D. iranica & Olea europaea & Oleaceae & Lamiales & Iran & Abdollazadeh et al. 2014 \\
\hline D. longicollis & $\begin{array}{l}\text { Lysiphyllum } \\
\text { cunninghamii }\end{array}$ & Fabaceae & Fabales & Australia & Pavlic et al. 2008 \\
\hline D. moneti & Acacia rostellifera & Fabaceae & Fabales & Australia & Taylor et al. 2009 \\
\hline D. neclivorem & Vitis vinifera & Vitaceae & Vitales & Australia & Pitt et al. 2015 \\
\hline \multirow[t]{2}{*}{ D. parva } & Corylus avellana, & Betulaceae & Fagales & Iran, Slovenia and Italy & Abdollazadeh et al. 2014, \\
\hline & Ostrya carpinifolia & Betulaceae & Fagales & Italy & Pavlic-Zupanc et al. 2015 \\
\hline D. pretoriensis & Acacia karroo & Fabaceae & Fabales & South Africa & Jami et al. 2012 \\
\hline D. prunicola & Prunus dulcis & Rosaceae & Rosales & Portugal & Abdollazadeh et al. 2014 \\
\hline D. santali & Santalum acuminatum & Santalaceae & Santalales & Australia & Taylor et al. 2009 \\
\hline \multirow[t]{6}{*}{ D. sarmentorum } & Acer platanoides & Sapindaceae & Sapindales & Poland & Mulenko et al. 2008 \\
\hline & Armeniaca vulgaris & Rosaceae & Rosales & Ukraine & Dudka et al. 2004 \\
\hline & Cornus sanguinea & Cornaceae & Cornales & Italy & Li et al. 2014 \\
\hline & Cornus sanguinea & Cornaceae & Cornales & Italy & This study \\
\hline & Coronilla emerus & Fabaceae & Fabales & Italy & This study \\
\hline & Crataegus sp & Rosaceae & Rosales & Italy & This study \\
\hline
\end{tabular}




\begin{tabular}{|c|c|c|c|c|c|}
\hline Dothiorella species & Host & Family & Order & Distribution & References \\
\hline & Euonymus europaeus & Celastraceae & Celastrales & Italy & This study \\
\hline & Malus pumila & Rosaceae & Rosales & Netherlands & Phillips et al. 2008, Wet et al. 2009, Chen et al. 2014 \\
\hline & Menispermum canadense & Menispermaceae & Ranunculales & Sweden & - \\
\hline & Paliurus spina-christi & Rhamnaceae & Rosales & Italy & This study \\
\hline & Persica vulgaris & Rosaceae & Rosales & Ukraine & Dudka et al. 2004 \\
\hline & Pistacia & Anacardiaceae & Sapindales & California & Chen et al. 2014 \\
\hline & Prunus americana & Rosaceae & Rosales & Florida & Perez et al. 2010 \\
\hline & Prunus domestica & Rosaceae & Rosales & Poland & Mulenko et al. 2008 \\
\hline & Prunus dulcis & Rosaceae & Rosales & California & Inderbitzin 2010, Lynch et al. 2013, Gramaje et al. 2012 \\
\hline & Prunus dulcis & Rosaceae & Rosales & Italy & This study \\
\hline & Prunus sp. & Rosaceae & Rosales & North America & Gramaje et al. 2012 \\
\hline & Prunus spinosa & Rosaceae & Rosales & Poland,Ukraine & Mulenko et al. 2008, Dudka et al. 2004 \\
\hline & Pyrus communis & Rosaceae & Rosales & Oregon, Washington & Luque et al. 2005 \\
\hline & Quercus ilex & Fagaceae & Fagales & ballota Spain & Sanchez et al. 2003 \\
\hline & Quercus suber & Fagaceae & Fagales & Spain & Sanchez et al. 2003 \\
\hline & Salix sp. & Salicaceae & Malpighiales & Italy & This study \\
\hline & Ulmus sp. & Ulmaceae & Rosales & England & Luque et al. 2005, Phillips et al. 2005 \\
\hline & Ulmus minor & Ulmaceae & Rosales & Italy & This study \\
\hline & Vitis vinifera & Vitaceae & Vitales & New Zealand & Baskarathevan et al. 2012 \\
\hline D. sempervirentis & Cupressus sempervirens & Cupressaceae & Pinales & Golestan, Iran & Abdollazadeh et al. 2014 \\
\hline D. striata & Citrus sinensis & Rutaceae & Sapindales & New Zealand & Abdollazadeh et al. 2014 \\
\hline \multirow[t]{4}{*}{ D. symphoricarposicola } & Symphoricarpos sp. & Caprifoliaceae & Dipsacales & Italy & Li et al. 2014 \\
\hline & Sambucus nigra & Adoxacea & Dipsacales & Italy & This study \\
\hline & Laurus nobilis & Lauraceae & Laurales & Italy & This study \\
\hline & Laburnum alpinum & Fabaceae & Fabales & Italy & This study \\
\hline D. thailandica & Bamboo & Poaceae & Poales & Thailand & Liu et al. 2012 \\
\hline D. thripsita & Acacia harpophylla & Fabaceae & Fabales & Australia & Shivas et al. 2009 \\
\hline D. ulmacea & Ulmus leavis & Ulmaceae & Rosales & Germany & Crous et al. 2015 \\
\hline D. uruguayensis & Hexachlamys edulis & Myrtaceae & Myrtales & Uruguay & Pérez et al. 2010 \\
\hline \multirow[t]{2}{*}{ D. vidmadera } & Vitis vinifera & Vitaceae & Vitales & Australia & Pitt et al. 2013 \\
\hline & Fraxinus ornus & Oleaceae & Lamiales & Italy & This study \\
\hline D. vinea-gemmae & Vitis vinifera & Vitaceae & Vitales & Australia & Pitt et al. 2015 \\
\hline
\end{tabular}


representing D. sarmentorum from Prunus dulcis, Salix sp. and Ulmus minor were collected. The other known reports of $D$. sarmentorum in above mentioned genera are Prunus dulcis (California), Salix sp. (Cyprus) and Ulmus sp. (UK). Dothiorella symphoricarposicola; a novel species which was recently introduced by $\mathrm{Li}$ et al. (2014) was isolated from Symphoricarpos sp. in Italy. Another three of our isolates from Sambucus nigra, Laurus nobilis and Laburnum alpinum clustered with D. symphoricarposicola (Fig. 1, table 2). The sexual morph of D. vidmadera was isolated from Fraxinus ornus, and the asexual morph of this species was isolated from Vitis vinifera in Australia (Pitt et al. 2013).

Eighteen Dothiorella species are known from only one host genus. Based on the information available; Dothiorella americana, D. brevicollis, D. casuarinae, D. dulcispinae, D. iranica, D. longicollis, D. moneti, D. neclivorem, D. pretoriensis, D. prunicola, D. santali, D. sempervirentis, D. striata, D. thailandica, D. thripsita, D. ulmacea, D. uruguayensis and D. vineagemmae species are limited to single host genera (Table 2). Although many Dothiorella species are host specific, many more collections are needed to confirm this. Dothiorella parva has been isolated from two host genera in a single host family Betulaceae, in the order Fagales, whereas D. vidmadera have been isolated from two host families; Vitaceae and Oleaceae (Table 2). Three Dothiorella species are known from a wide range of host genera or families. Dothiorella iberica, D. sarmentorum and D. symphoricarposicola occur across many host families and orders. Dothiorella iberica has been isolated from 12 host genera (from 6 host families), whereas D. sarmentorum has been isolated from 17 host genera (from 12 host families), while D. symphoricarposicola has been isolated from four host genera in four different host families. The information up to date suggests that most Dothiorella species have narrow host ranges, while a few species have very wide host ranges. As more hosts from diverse continents are studied, we should expect many more species to be discovered in this genus.

Our study illustrates the diversity of Dothiorella species associated with twig and branches of woody hosts. Four species were identified. Dothiorella sarmentorum was the dominant species (65\% of isolates), followed by D. symphoricarposicola (20\%), D. iberica (8\%) and D. vidmadera $(8 \%)$. All studied Dothiorella species occurred on more than one host, indicating that they are not host-specific, but host-generalists.

\section{Acknowledgement}

The research was supported by Innovation Funds of IPEP, Beijing Academy of Agriculture and Forestry Sciences and CARS-30 China. We gratefully acknowledge The Mushroom Research Foundation, Chiang Rai, Thailand; K.D. Hyde thanks the Chinese Academy of Sciences, project number 2013T2S0030, for the award of Visiting Professorship for Senior International Scientists at Kunming Institute of Botany.

\section{References}

Abdollahzadeh J, Javadi A, Zare R, Phillips AJL. 2014 - A phylogenetic study of Dothiorella and Spencermartinsia species associated with woody plants in Iran, New Zealand, Portugal and Spain. Persoonia 32, 1-12.

Adesemoye AO, Mayorquin JS, Wang DH, Twizeyimana M, Lynch SC, Eskalen A. 2014 Identification of Species of Botryosphaeriaceae Causing Bot Gummosis in Citrus in California. Plant Disease 98, 55-61.

Alves A, Barradas C, Phillips AJL, Correia A. 2013 - Diversity of Botryosphaeriaceae species associated with conifers in Portugal. European Journal of Plant Pathology 135, 791-804.

Armaleo D, Clerc P. 1995 - A rapid and inexpensive method for the purification of DNA from lichens and their symbionts. Lichenologists 27, 207-213.

Azouaoui-Idjer G, Rocca GD, Pecchioli A, Bouznad Z, Danti R. 2012 - First report of Botryosphaeria iberica associated with dieback and tree mortality of Monterey Cypress (Cupressus macrocarpa) in Algeria. Plant Disease 96, 1073. 
Baskarathevan J, Jaspers MV, Ridgway HJ, Jones E. 2012 - Incidence and distribution of botryosphaeriaceous species in New Zealand vineyards. European Journal of Plant Pathology 132, 549-560.

Burgess TI, Barber PA, Mohali S, Pegg G, de Beer W, Wingfield MJ. 2006 - Three new Lasiodiplodia spp. from the tropics, recognized based on DNA sequence comparisons and morphology. Mycologia 98, 423-435.

Carbone I, Kohn LM. 1999 - A method for designing primer sets for speciation studies in filamentous Ascomycetes. Mycologia 91, 553-556.

Chen SF, Morgan DP, Michailides TJ. 2014 - Botryosphaeriaceae and Diaporthaceae associated with panicle and shoot blight of pistachio in California, USA. Fungal Diversity 67, 157-179.

Chomnunti P, Hongsanan S, Aguirre-Hudson B, Tian Q, Peršoh D, Dhami MK, Alias AS, Xu J, Liu Z, Stadler M, Hyde KD. 2014 - The sooty moulds. Fungal Diversity 66, 1-36.

Crous PW, Palm ME. 1999 - Reassessment of the anamorph genera Botryodiplodia, Dothiorella and Fusicoccum. Sydowia 51, 167-175.

Crous PW, Schumacher RK, Wingfield MJ, Lombard L, Giraldo A, Christensen M, Gardiennet A, Nakashima C, Pereira OL, Smith AJ, Groenewald JZ. 2015 - Fungal Systematics and Evolution: FUSE 1. Sydowia 67, 81-118.

Crous PW, Slippers B, Wingfield MJ, Rheeder J, Marasas WFO, et al. 2006 - Phylogenetic lineages in the Botryosphaeriaceae. Studies in Mycology 55, 235-253.

Dissanayake AJ, Zhang W, Li XH, Zhou Y, Chethana KWT, Chukeatirote E, Hyde KD, Yan JY, Zhang G, Zhao S. 2015 - First report of Neofusicoccum mangiferae associated with grapevine dieback in China. Phytopathologia Mediterranea 54, 414-419.

Doll DA, Rolshausen PE, Pouzoulet J, Michailides TJ. 2015 - First report of Dothiorella iberica causing trunk and scaffold cankers of almond in California Plant Disease. 99, 1185.

Dudka IO, Heluta VP, Tykhonenko YY, Andrianova TV, Hayova VP, Prydiuk MP, Dzhagan VV, Isikov VP. 2004 - Fungi of the Crimean Peninsula. M.G. Kholodny Institute of Botany, National Academy of Sciences of Ukraine, 452 pages.

Gramaje D, Agusti-Brisach C, Perez-Sierra A, Moralejo E, Olmo D, Mostert L, Damm U, Armengol J. 2012 - Fungal trunk pathogens associated with wood decay of almond trees on Mallorca (Spain). Persoonia 28, 1-13.

Hall T. 2006 - Bioedit 7.5.0.3. Department of Microbiology, North Carolina State University. http://www.mbio.ncsu.edu/BioEdit/Bioedit.html.

Hillis DM, Bull JJ. 1993 - An empirical test of bootstrapping as a method for assessing confidence in phylogenetic analysis. Systamatic Biology 42, 182-192.

Hyde KD, Jones EGB, Liu JK, Ariyawansa H, Boehm E, Boonmee S, Braun U, Chomnunti P, Crous PW, Dai DQ, Diederich P, Dissanayake A, Doilom M, Doveri F, Hongsanan S, Jayawardena R, Lawrey JD, Li YM, Liu YX, Lücking R, Monkai J, Muggia L, Nelsen MP, Pang KL, Phookamsak R, Senanayake IC, Shearer CA, Suetrong S, Tanaka K, Thambugala KM, Wijayawardene NN, Wikee S, Wu HX, Zhang Y, Aguirre HB, Alias SA, Aptroot A, Bahkali AH, Bezerra JL, Bhat DJ, Camporesi E, Chukeatirote E, Gueidan C, Hawksworth DL, Hirayama K, Hoog S. de, Kang JC, Knudsen K, Li WJ, Li XH, Liu ZY, Mapook A, Mckenzie EHC, Miller AN, Mortimer PE, Phillips AJL, Raja HA, Scheuer C, Schumm F, Taylor JE, Tian Q, Tibpromma S, Wanasinghe DN, Wang Y, Xu JC, Yacharoen S, Yan JY, Zhang M. 2013 - Families of Dothideomycetes. Fungal Diversity 63, 1-313.

Hyde KD, Nilsson RH, Alias SA, Ariyawansa HA, Blair JE, Cai L, de Cock AWAM, Dissanayake AJ, Glockling SL, Goonasekara ID, Gorczak M, Hahn M, Jayawardena RS, van Kan JAL, Laurence MH, Lévesque CA, Li XH, Liu JK, Maharachchikumbura SSN, Manamgoda DS, Martin FN, McKenzie EHC, McTaggart AR, Mortimer PE, Nair PVR, Pawłowska J, Rintoul TL, Shivas RG, Spies CFJ, Summerell BA, Taylor PWJ, Terhem RB, Udayanga D, Vaghefi N, Walther G, Wilk M, Wrzosek M, Xu JC, Yan JY, Zhou N. 2014 - One stop shop: backbones trees for important phytopathogenic genera: I. Fungal Divers 67, 21-125. 
Inderbitzin P, Bostock RM, Trouillas FP, Michailides TJ. 2010 - A six locus phylogeny reveals high species diversity in Botryosphaeriaceae from California almond. Mycologia 102, 13501368.

Jami F, Slippers B, Wingfield M J. 2012 - Five new species of the Botryosphaeriaceae from Acacia karroo in South Africa Cryptogamie Mycologie 33, 245-266.

Katoh K, Toh H. 2008 - Recent developments in the MAFFT multiple sequence alignment program. Briefings in Bioinformatics 9, 276-285.

Li W, Liu JK, Bhat DJ, Camporesi E, Xu J, Hyde KD. 2014 - Introducing the novel species, Dothiorella symphoricarposicola, from snowberry in Italy. Cryptogamie Mycologie 35, 257270.

Liu JK, Phookamsak R, Mingkhuan M, Wikee S, Li YM, Ariyawansha H, Boonmee S, Chomnunti P, Dai DQ, Bhat JD, Romero AI, Zhuang WY, Monkai J, Gareth Jones EB, Chukeatirote E, Ko Ko TW, Zhao YC, Wang Y, Hyde KD. 2012 - Towards a natural classification of Botryosphaeriales. Fungal Divers 57: 149-210.

Luque J, Martos S, Phillips AJL. 2005 - Botryosphaeria viticola sp. nov. on grapevines: a new species with a Dothiorella anamorph. Mycologia 97, 1111-1121.

Lynch SC, Eskalen A, Zambino PJ, Mayorquin JS, Wang DH. 2013 - Identification and pathogenicity of Botryosphaeriaceae species associated with coast live oak (Quercus agrifolia) decline in southern California. Mycologia 105, 125-140.

Lynch SC, Zambino PJ, Scott TA, Eskalen A. 2014 - Occurrence, incidence and associations among fungal pathogens and Agrilus auroguttatus, and their roles in Quercus agrifolia decline in California. Forest Pathology 44, 62-74.

McDonald V, Eskalen A. 2011 - Botrysphaeriaceae species associated with avacado branch cankers in California. Pl. Dis. 95, 1465-1473.

Mulenko W, Majewski T, Ruszkiewicz-Michalska M. 2008 - A Preliminary Checklist of Micromycetes in Poland. W. Szafer Institute of Botany, Polish Academy of Sciences 9, 752.

Nylander JAA. 2004 - MrModeltest v2. Program distributed by the author. Evolutionary Biology Centre, Uppsala University, Sweden.

Page RD. 1996 - TreeView: an application to display phylogenetic trees on personal computers. Computer Applications in the Biosciences 12, 357-358.

Pavlic D, Wingfield MJ, Barber B, Slippers B, Hardy GEJ, Burgess TI. 2008 - Seven new species of the Botryosphaeriaceae from baobab and other native trees in Western Australia. Mycologia 100, 851-866.

Pavlic-Zupanc D, Piskur B, Slippers B. 2015 - Molecular and morphological characterization of Dothiorella species associated with dieback of Ostrya carpinifolia in Slovenia and Italy.Phytopathologia Mediterranea 54, 222-231.

Pérez CA, Wingfield MJ, Slippers B, Altier NA, Blanchette RA. 2010 - Endophytic and cankerassociated Botryosphaeriaceae occurring on non-native Eucalyptus and native Myrtaceae trees in Uruguay. Fungal Diversity 41, 53-69.

Phillips A, Alves A, Correia A, Luque J. 2005 - Two new species of Botryosphaeria with brown, 1septate ascospores and Dothiorella anamorphs. Mycologia 97, 513-529.

Phillips AJL, Alves A, Abdollahzadeh J, Slippers B, Wingfield MJ, Groenewald JZ, Crous PW. 2013 - The Botryosphaeriaceae: genera and species known from culture. Studies in Mycology 76, 51-167.

Phillips AJL, Alves A, Pennycook SR, Johnston PR, Ramaley A, Akulov A, Crous PW. 2008 Resolving the phylogenetic and taxonomic status of dark-spored teleomorph genera in the Botryosphaeriaceae. Persoonia 21, 29-55.

Pitt WM, Úrbez-Torres JR, Trouillas FP. 2013 - Dothiorella vidmadera, a novel species from grapevines in Australia and notes on Spencermartinsia. Fungal Diversity 61, 209-219.

Pitt WM, Úrbez-Torres JR, Trouillas FP. 2015 - Dothiorella and Spencermartinsia, new species and records from grapevines in Australia. Australasian Plant Pathology 44, 43-56. 
Qiu Y, Steel CC, Ash GJ, Savocchia S. 2011 - Survey of Botryosphaeriaceae associated with grapevine decline in the Hunter Valley and Mudgee grape growing regions of New South Wales. Australasian Plant Pathology 40, 1-11.

Rodríguez F, Oliver JL, Marín A, Medina JR. 1990 - The general stochastic model of nucleotide substitution. Journal of Theoretical Biology 142, 485-501.

Ronquist FR, Huelsenbeck JP. 2003 - MrBayes3: Bayesian phylogenetic inference under mixed models. Bioinformatics 19, 1572-1574.

Sanchez ME, Venegas J, Romero MA, Phillips AJL, Trapero A. 2003 - Botryosphaeria and related taxa causing oak canker in south western Spain. Plant Disease 87, 1515-1521.

Shivas RG, Tree DJ, Tan YP, Ballard EL. 2009 - Dothiorella thripsita. Fungal Planet 32, Persoonia 22, 168-169.

Sivanesan A. 1984 - The bitunicate Ascomycetes and their anamorphs. Vaduz, Liechtenstein: J Cramer.

Slippers B, Roux J, Wingfield MJ, van der Walt, FJJ, Jami F, Mehl JWM, Marais GJ. 2014 Confronting the constraints of morphological taxonomy in the Botryosphaeriales. Persoonia $33,55-168$.

Sutton BC. 1977 - Coelomycetes. VI. Nomenclature of generic names proposed for Coelomycetes. Mycological Papers 141, 1-253.

Swofford DL. 2003 - PAUP*. Phylogenetic analysis using parsimony (*and other methods). Version 4.0. Sunderland, Massachusetts, Sinauer Associates.

Taylor K, Barber PA, St J Hardy GE, Burgess TI. 2009 - Botryosphaeriaceae from tuart (Eucalyptus gomphocephala) woodland, including descriptions of four new species. Mycological Research 113, 337-353.

Twizeyimana M, McDonald V, Mayorquin JS. 2013 - Effect of Fungicide Application on the Management of Avocado Branch Canker (Formerly Dothiorella Canker) in California Plant Disease 97, 897-902.

Úrbez-Torres JR, Gubler WD, Luque J. 2007 - First Report of Botryosphaeria iberica and B. viticola associated with grapevine decline in California. Plant Disease 91, 772.

Úrbez-Torres JR, Peduto F, Striegler RK. 2012 - Characterization of fungal pathogens associated with grapevine trunk diseases in Arkansas and Missouri Fungal Diversity 52, 169-189.

Úrbez-Torres JR, Peduto F, Vossen PM, Krueger WH, Gubler WD. 2013 - Olive Twig and Branch Dieback: Etiology, incidence, and distribution in California. Plant Disease 97, 231-244.

Wet J de, Slippers B, Preisig O, Wingfield BD, Tsopelas P, Wingfield MJ. 2009 - Molecular and morphological characterization of Dothiorella casuarini sp. nov. and other Botryosphaeriaceae with diplodia-like conidia. Mycologia 101, 503-511.

White TJ, Bruns T, Lee S, Taylor J. 1990 - Amplified and direct sequencing of fungal ribosomal RNA genes for phylogenies. In: Innis MA, Gelfand DH, Sninsky JJ, White TJ (eds) PCR protocols: a guide to methods and applications. Academic, San Diego, pp 315-322.

Wijayawardene NN, Crous PW, Kirk PM, Hawksworth DL, Boonmee S, Braun U, Dai DQ, D'souza MJ, Diederich P, Dissanayake AJ, Raja HA, Shivas RG, Slippers B, Taylor JE, Tian Q, Wang Y, Woudenberg JHC, Cai L, Jaklitsch WM, Hyde KD. 2014 - Naming and outline of Dothideomycetes-2014 including proposals for the protection or suppression of generic names. Fungal Diversity 69, 1-55.

Zhou JP, Lu Q, Liang J, Decock C, Zhang XY. 2010 - Lasiodiplodia pseudotheobromae, a new record of pathogenic fungus from some subtropical and tropical trees in southern China. Cryptogamie Mycologie 31, 431-439. 